\title{
The safety of oral fluconazole therapy in pregnancy
}

\author{
Vanessa Cristine Paquette BSc(Pharm) PharmD, Chelsea Elwood MD
}

Cite as: CMAJ 2019 February 19;191:E177-8. doi: 10.1503/cmaj.190079

See related article at www.cmaj.ca/lookup/doi/10.1503/cmaj.180963

$\mathbf{V}$ ulvovaginal candidiasis affects up to $75 \%$ of women during their lifetime and disproportionately affects pregnant women. It has not been associated with adverse pregnancy outcomes, and treatment of symptomatic infection is recommended to alleviate discomfort. ${ }^{1}$ During pregnancy, because of uncertainties regarding safety of systemic fluconazole, most guidelines recommend first-line treatment with a topical imidazole and caution against the use of oral fluconazole in pregnancy. ${ }^{2,3}$ This warning stems from associations with use of fluconazole in the first trimester and spontaneous abortions and major congenital malformations.

In a linked paper, Bérard and colleagues assess the effect of exposure to low- and high-dose fluconazole during pregnancy on the occurrence of spontaneous abortions, major congenital malformations and stillbirths, by conducting 3 nested case-control studies, using data from the Quebec Pregnancy Cohort between 1998 and 2015. ${ }^{4}$ There were 320868 pregnancies included in an analysis of spontaneous abortions, 226599 in an analysis of major congenital malformations, and 7832 in an analysis of stillbirths. Adjusting for potential confounders, use of low-dose fluconazole ( $<150 \mathrm{mg}$ ) and high-dose fluconazole (> $150 \mathrm{mg}$ ) was associated with an increased risk of spontaneous abortion (adjusted odds ratio [OR] for 345 cases exposed to low-dose treatment 2.23, 95\% confidence interval $[\mathrm{Cl}]$ 1.96-2.54; adjusted OR for 249 cases exposed to high-dose treatment $3.20,95 \% \mathrm{Cl} 2.73-3.75$ ). Exposure to low-dose fluconazole during the first trimester did not increase the risk of major congenital malformations. Exposure to high-dose fluconazole during the first trimester was associated with an increased risk of anomalies of cardiac septal closure (OR 1.81, 95\% Cl 1.04-3.14; 13 exposed cases), but this should be interpreted with caution owing to the low number of exposed cases. No association was found between any dose of fluconazole and stillbirth.

These findings are consistent with previously published literature. In a registry cohort study from Denmark, there was a significantly increased risk of spontaneous abortion associated with exposure to fluconazole between 7 and 22 weeks' gestation (hazard ratio [HR] 1.48; 95\% Cl, 1.23-1.77). There was no significant association between exposure to fluconazole and stillbirth (HR 1.32 [95\% $\mathrm{Cl} 0.82-2.14])$. To test for confounding by indication, preg-

\section{KEY POINTS}

- Vulvovaginal candidiasis affects up to $75 \%$ of women during their lifetime and disproportionately affects pregnant women.

- Accumulated evidence suggests that use of low-dose oral fluconazole in pregnancy is associated with spontaneous abortions but not congenital malformations or stillbirth.

- Topical azoles, rather than low-dose fluconazole, should be first-line treatment for vulvovaginal candidiasis in early pregnancy.

nancies exposed to intravaginal formulations of topical azoles were used as an additional comparator group, yielding similar results with an increased risk of spontaneous abortion with exposure to fluconazole (HR 1.62 [95\% Cl 1.26-2.07]) but not stillbirth (HR $1.18[95 \% \mathrm{Cl}, 0.64-2.16]) .^{5}$ The results of this trial prompted the US Food and Drug Administration and Health Canada to conduct safety reviews and release advisories on use of low-dose fluconazole in pregnancy. The manufacturer of Diflucan One (fluconazole $150 \mathrm{mg}$ ), the Canadian over-the-counter product, voluntarily updated its product safety information. Health Canada noted that while the results of this study are not definitive, it agreed with updating the safety information and requested that all available over-the-counter products follow suit. ${ }^{6}$

In case reports, exposure to high-dose fluconazole (400 to $800 \mathrm{mg}$ per day) during the first trimester of pregnancy increased the risk of congenital malformations resembling Antley-Bixler syndrome. ${ }^{7,8}$ The risk of congenital malformations with exposure to low-dose fluconazole seems to be minimal, if there is a risk at all. A 2015 systematic review and meta-analysis included 4 studies examining the risk of congenital malformations in offspring of women exposed to fluconazole in the first trimester of pregnancy. ${ }^{9}$ The rate for overall malformations was $1.10(95 \% \mathrm{Cl}$ 0.98-1.25), heart defects was 1.29 (95\% Cl 1.05-1.58), craniofacial defects was 1.25 (95\% Cl 0.88-1.77), and limb or musculoskeletal defects was 0.82 (95\% Cl 0.59-1.13). The study's authors concluded that the use of fluconazole in the first trimester does not appear to increase the overall risk for congenital malformations. 
A 2016 study that used data from the National Birth Defects Prevention Study, with data from 1997 onward, found that use of fluconazole in the first trimester was significantly associated with cleft lip with cleft palate (OR 5.53, 95\% Cl 1.68-18.24) and with transposition of the great arteries (OR 7.56, 95\% Cl 1.22-35.45). ${ }^{10}$ However, despite the large size of the study, a small number of mothers were reportedly taking fluconazole in the first trimester. There was a small number of exposed infants with each birth defect, and only 6 exposed control infants. Furthermore, information on fluconazole dosage was not collected.

All studies come with limitations and the linked study uses prescription databases as a source of medication exposure, which documents prescriptions filled, rather than actual prescriptions taken. It is also unclear whether patients may have been started on systemic fluconazole in hospital for a more invasive fungal infection and then discharged into the community to complete therapy. If cumulative dosing rendered a patient into exposure to low- versus high-dose fluconazole, then true total exposure may not have been captured. The authors do not address fluconazole exposure as an over-the-counter product. A key piece of information is missing; i.e., the type of fungal infection, as mycoses were reported together as 1 group.

Despite these limitations, this study validates what is already known about the use of oral fluconazole in pregnancy: it may be associated with spontaneous abortion but not stillbirth. In addition, exposure to low-dose fluconazole is unlikely to be associated with congenital malformations. The study re-emphasizes safe prescribing practices in pregnancy, which include confirming the correct diagnosis and then choosing the safest medication with the largest body of data in pregnancy at the lowest appropriate doses. For women with vulvovaginal candidiasis, topical azoles should be the treatment of choice. ${ }^{2,3}$ For women with disseminated fungal disease, all treatment risks should be considered in the context of the health of the mother. If treatment is indicated, a risk-benefit discussion should take place, but appropriate treatment should not be deferred if indicated. A healthy mom is required for a healthy baby.

\section{References}

1. Young GL, Jewell D. Topical treatment for vaginal candidiasis (thrush) in pregnancy. Cochrane Database Syst Rev 2001;(4):CD000225.

2. van Schalkwyk J, Yudin MH; Infectious Disease Committee. Vulvovaginitis: screening for and management of trichomoniasis, vulvovaginal candidiasis, and bacterial vaginosis. J Obstet Gynaecol Can 2015;37:266-74.

3. Vulvovaginal candidiasis. Atlanta: Centers for Disease Control and Prevention; reviewed 2015 June 4. Available: www.cdc.gov/std/tg2015/candidiasis.htm (accessed 2019 Jan. 10).

4. Bérard A, Sheehy O, Zhao J-P, et al. Associations between low- and high-dose oral fluconazole and pregnancy outcomes: 3 nested case-control studies. CMAJ 2019;191:E179-87.

5. Mølgaard-Nielsen D, Svanström H, Melbye M, et al. Association between use of oral fluconazole during pregnancy and risk of spontaneous abortion and stillbirth. JAMA 2016;315:58-67.

6. The summary safety review - non-prescription fluconazole - assessing potential risks to pregnancy outcomes. Ottawa: Health Canada; 2017. Available: www.canada.ca/en/health-canada/services/drugs-health-products/medeffect -canada/safety-reviews/non-prescription-fluconazole-assessing-potential-risks -pregnancy-outcomes.html (accessed 2019 Jan. 10).

7. Aleck KA, Bartley DL. Multiple malformation syndrome following fluconazole use in pregnancy: report of an additional patient. Am J Med Genet 1997;72:253-6.

8. Lopez-Rangel E, Van Allen MI. Prenatal exposure to fluconazole: an identifiable dysmorphic phenotype. Birth Defects Res A Clin Mol Teratol 2005;73:919-23.

9. Alsaad AM, Kaplan YC, Koren G. Exposure to fluconazole and risk of congenital malformations in the offspring: a systematic review and meta-analysis. Reprod Toxicol 2015;52:78-82.

10. Howley MM, Carter TC, Browne ML, et al. National Birth Defects Prevention Study. Fluconazole use and birth defects in the National Birth Defects Prevention Study. Am J Obstet Gynecol 2016;214:657.e1-9.

\section{Competing interests: None declared.}

This article was solicited and has not been peer reviewed.

Affiliations: Department of Pharmacy (Paquette), Department of Obstetrics and Gynecology (Elwood), British Columbia Women's Hospital and Health Centre; Department of Obstetrics and Gynecology (Elwood), University of British Columbia; Women's Health Research Institute (Elwood), Vancouver, BC
Contributors: Both authors contributed to the conception and design of the work. Vanessa Paquette drafted the manuscript. Both authors revised it critically for important intellectual content, gave final approval of the version to be published and agreed to be accountable for all aspects of the work.

Correspondence to: Vanessa Paquette,vpaquette@phsa.ca 